\title{
Design Considerations by the Optimization of PMS Motors
}

\author{
B. Kollár
}

\author{
Széchenyi István University, Department of Automation \\ Research Center of Vehicle Industry \\ Egyetem tér 1, H-9026 Győr, Hungary \\ e-mail: kollar.bettina@sze.hu
}

\begin{abstract}
The determination of the targets, the input, implicit and design parameter is very important part in the design optimization of the permanent magnetic synchronous (PMS) motors for electric vehicles. The connections were defined between them, investigations were made which assisted to the appropriate optimization. In this paper the researches for the design optimization are presented which define the targets correctly and describes the results of the calculations and simulations of the slot fill factor.
\end{abstract}

Keywords: PMS motor, optimization, demagnetisation, slot fill factor, penalty function, electric vehicle

\section{Introduction}

It is important to aim to find the optimum of the specified parameters by the design of electric motors. Usually the target of the optimization is to minimise the losses.

The objectives like minimum building size, total cost, induced voltage, demagnetisation and size of the permanent magnets will be important too if the electric motor will drive a vehicle [1-4].

In this paper those design methods will be introduced which were necessary to execute the optimization of permanent magnetic synchronous motors.

\section{The model of the PMS motor}

Permanent magnetic synchronous motor was used with outer rotor construction during the design and optimization process. The motor will drive vehicle so the design parameters were planned along these lines. The first input of the process is the required driving cycle. The operating points of the vehicle are determined from the driving cycle in view of the mass of the vehicle, the transmission and the diameter of the wheels [6]. In addition the maximum outer diameter of the rotor is knew before the design process which imply the building size of the motor.

In this work the finite element software ANSYS Maxwell is used to design, calculate and simulate the models of the motor. The model of the motor is shown in Fig. 2. The 
Design and Optimization Toolbox of Matlab is used to makes the optimization process with multi-objective genetic algorithm. The design parameters of the optimization are illustrated in Fig. 1:

- $\quad$ tooth gap width $\left(\mathrm{B}_{\mathrm{s} 0}\right)$

- tooth tang depth $\left(\mathrm{H}_{\mathrm{s} 0}\right)$

- $\quad$ slot depth $\left(\mathrm{H}_{\mathrm{s} 2}\right)$

- air gap thickness

- length of the stator

- magnet gap

- magnet thickness

- number of turns

- wire diameter

- tooth width (f)

- rotor outer diameter

- stator inner diameter

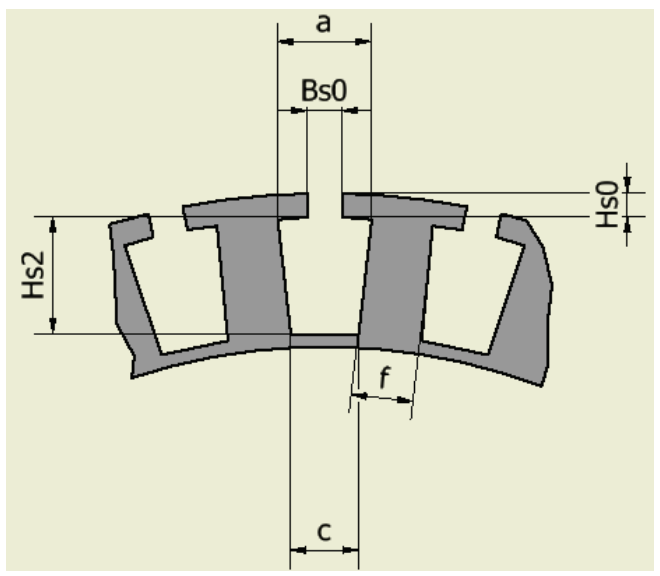

Figure 1. Sizes of the slot

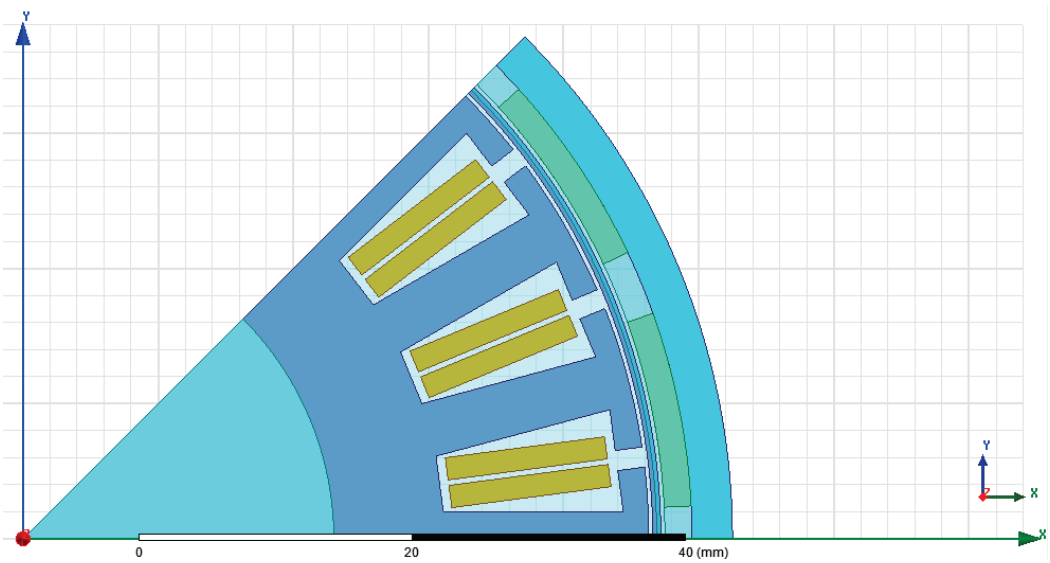




\section{Figure 2. The 2D model of the motor-segment in ANSYS Maxwell}

Lower and upper limit values were given to these parameters. The rotor outer diameter was handled like an input of the optimization as the upper limit value. The teeth of the stator are parallel with each other because of the smaller cogging torque [12].

The next list includes the implicit parameters which were determined from the design parameters:

- Wires of conductor

- Slot width in the upper part (a)

- Slot width in the lower part (c)

- Rotor inner diameter

- Stator outer diameter

- Slot fill factor

- $\quad$ Tooth gap width $\left(\mathrm{H}_{\mathrm{s} 0}\right)$

The connections between the parameters are determined with equations, for example the winding size could not be bigger than the slot.

The targets of the optimization were to minimize the total losses, the weight of the motor and minimize the amount of the penalty functions. The calculation of the total loss was given by the next equations. The core loss $\left(\mathrm{p}_{\mathrm{c}}\right)$ is calculated from the amount of the hysteresis loss and the eddy current loss $[5,6]$.

$$
\mathrm{p}_{\mathrm{c}}=\mathrm{K}_{\mathrm{h}} \mathrm{f}|\mathrm{B}|^{2}+\mathrm{K}_{\mathrm{e}}(\mathrm{f}|\mathrm{B}|)^{2}
$$

where $K_{h}$ is the hysteresis loss constant, $K_{e}$ is the eddy current loss constant, $B$ is the magnetic flux density and $f$ is the sinusoidal varying frequency of $B$. The value of $\mathrm{p}_{\mathrm{c}}$ is calculated by ANSYS Maxwell. $\mathrm{K}_{\mathrm{h}}$ and $\mathrm{K}_{\mathrm{e}}$ are the parameters of the chosen material in the software.

The winding loss was calculated according to this equation:

$$
P_{w}=3\left(\frac{I^{2}}{\sqrt{2}}\right) R_{w} l N p
$$

where $I$ is the amplitude of the phase current, $R_{w}$ is the resistance of the wire, $l$ is the length of the stator, $N$ is the number of the turns, $p$ is the pole number of the motor.

The summarized loss is [6]:

$$
\mathrm{P}_{\text {sum }}=\mathrm{P}_{\mathrm{w}}+\int_{\mathrm{V}} \mathrm{p}_{\mathrm{c}}
$$

\section{Determination of the penalty functions}

The results of a multi-objective optimization can be displayed graphically more beneficial in $2 \mathrm{D}$ if the number of the objectives are not higher than 3 [1]. For this the third objective of the optimization is to minimize the sum of the torque ripple and the demagnetisation. 
These parameters were defined with penalty functions which can handle the limits of the parameters and can give penalty when the value is higher than the specified limit. These functions are the logistic functions $(\mathrm{P}(\mathrm{t}))$ which is shown in Fig. 3.

$$
P(t)=\frac{1}{1+e^{-t}}
$$

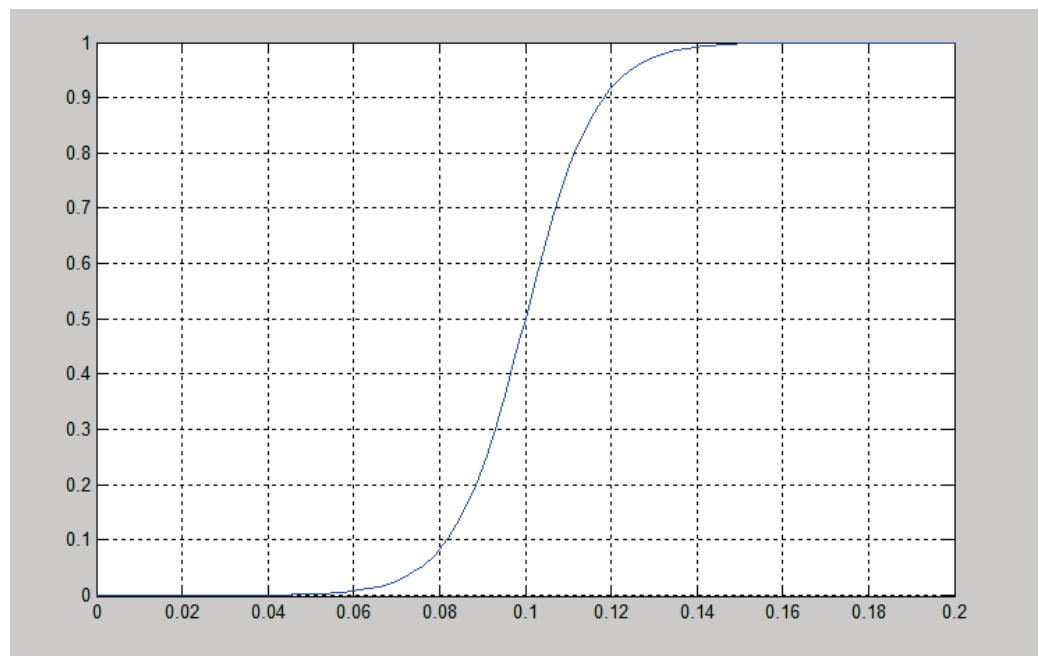

Figure 3. The logistic function

The limits were appointed by the definition of the penalty functions, the first is where the value of the given parameter is good (the penalty is close to 0 ) the other is where the value of the parameter is absolute incorrect (the penalty is close to 1).

\subsection{The penalty function of the cogging torque}

The torque of the motor is created by the interaction of the current which flows in the winding and the flux density distribution which was generated by the permanent magnetic rotor. As the flux density distribution and the stator currents change in time the torque which is constant in time is ensured for the motor, these were fitted to each other that the product of them is permanent. If it does not come into existence perfect there will be cogging torque.

The cogging torque has vibration induced effect to the mechanical elements of the vehicles so it is necessary to keep it under a determined limit [12]. The two limits of the logistic function are defined by the experience of the motor design and the literature research to $2,5 \%$ and $5 \%$.

\subsection{The penalty function of the demagnetization}

The demagnetization curve is the part of the hysteresis loop curve where the magnetic flux density (B) is positive and the magnetic field intensity $(\mathrm{H})$ is negative. This curve is important because the operation point of the built-in magnetized permanent magnet moves in the demagnetisation part of the hysteresis loop. 


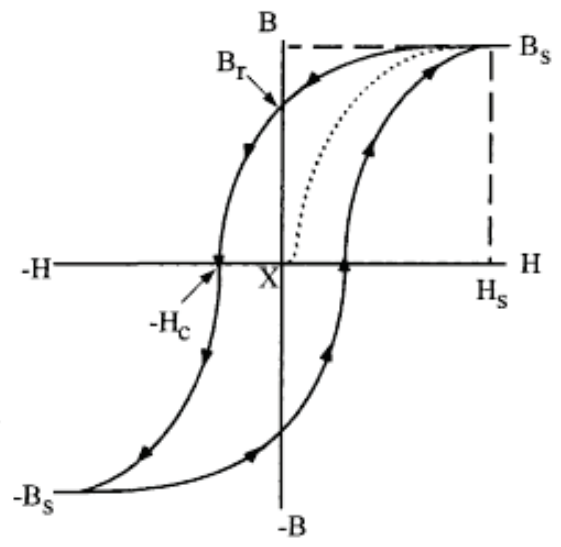

Figure 4. Hysteresis loop [7]

One of the important points is the remanent magnetic flux density (Br). It is the magnetic flux density which corresponds to zero field intensity. If it increases the flux and the inducted voltage of the motor get larger. The other point is the coercive field strength $(\mathrm{Hc})$ which releases the magnetic flux density to zero in the open-loop magnetic circle permanent magnet $[8,9,13]$.

The remanent magnetic flux density and the coercive field strength are temperaturedependent values. If the temperature of the magnet transcended the specified limit the operation point of the magnet can slide down from the linear part of the demagnetization curve. After that the original magnetic flux density is not insured to get back to room temperature only if it will be magnetise again. The target is avoid the work in the linear part.

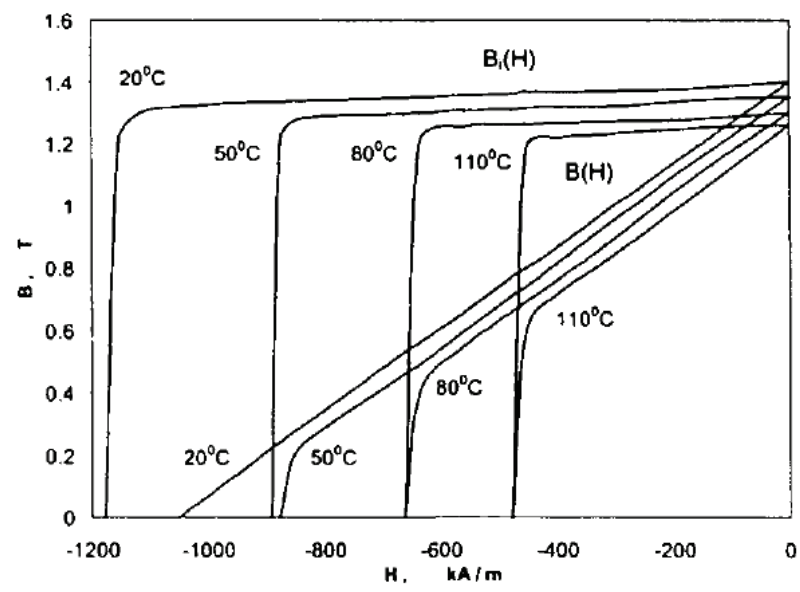

Figure 5. Demagnetisation curves and their variations with the temperature for sintered $\mathrm{NdFeB} \mathrm{[9]}$ 
The maximum operation temperature was specified like an input parameter of the optimization. Thereto the insulation class of the winding materials was considered and proportion the permanent magnet to this temperature.

The temperature-dependence can be chosen of the magnet material in ANSYS Maxwell. These data are in the catalogue of the magnet factories. These parameters are the temperature coefficient of the remanent magnetic flux density $\left(\mathrm{RTC}_{\mathrm{Br}}\right)$ and the temperature coefficient of the coercitive field strength $\left(\mathrm{RTC}_{\mathrm{Hc}}\right)[8,9,13]$.

$$
B_{r}(T)=B_{r 20}\left(1-\frac{R T C_{B r}(T-20)}{100}\right)
$$

where $B_{r}(T)$ is the remanent magnetic flux density in the specified maximum operating temperature, $B_{r 20}$ the remanent flux density in room temperature (catalogue data), $T$ is the specified maximum working temperature [9].

$$
\mathrm{H}_{\mathrm{c}}(\mathrm{T})=\mathrm{H}_{\mathrm{c} 20}\left(1-\frac{\mathrm{RTC}_{\mathrm{Hc}}(\mathrm{T}-20)}{100}\right)
$$

where $H_{c}(T)$ is the coercive field strength in the specified maximum working temperature, $H_{c 20}$ is the coercive field strength in room temperature (catalogue data) [9].

The values of the remanent magnetic flux density and the coercive field strength in the maximum operating temperature should be considered at the same time. So the optimizer has to give penalty to the demagnetisation to the member of the optimization if the $\mathrm{B}_{\mathrm{r}}(\mathrm{T})$ is higher and $\mathrm{H}_{\mathrm{c}}(\mathrm{T})$ is lower than the calculated values.

\section{Simulations to determinate the slot fill factor}

Currently the slots were handle with double layer winding. It reduces the cogging torque of the motor [10]. The insulation class of the winding materials are $\mathrm{H}\left(180^{\circ} \mathrm{C}\right)$. During the reeling a slot liner is placed first in the slots, which thickness is $0.31 \mathrm{~mm}$. The slots are closed with a slot wedge which is usually from bakelite material by this shape of slots. The thickness of it is $1 \mathrm{~mm}$ and it is placed under the tooth tang. The shape of the slots is trapeze, like in the Fig. 1. The sizes of the slot (a, c, Hs2) change because of the slot liners and slot wedge. This area is calculated for each of the optimization's member.

$$
T_{\text {slotV }}=\frac{a-(2 \cdot 0.31)+c-(2 \cdot 0.31)}{2}\left(H_{s 2}-(2 \cdot 0.31+1)\right)
$$

Round enamelled copper wires are used which are placed in the slots like in the schematic drawing in Fig. 6. When the wires are placed next to each others there are losses which are air. So a round wire occupies a hexagonal area in the slot.

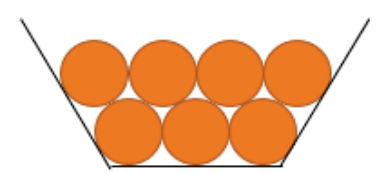

Figure 6. Round copper wires in the slot 
In theory the slot fill factor can be better with wires with smaller diameter. Smaller current can flow across the smaller diameter wire because the resistance is bigger, so the winding losses will be bigger too. Therefore the software controls the winding losses according to the eq. (2) after it chose the size of the wire.

Table 1. The data of the wires [11]

\begin{tabular}{|c|c|c|}
\hline Effective diameter & Enamelled diameter & $\begin{array}{c}\text { DC resistance at 20 } \\
{ }^{\circ} \boldsymbol{C}[\boldsymbol{\Omega} / \mathbf{m}]\end{array}$ \\
\hline 0.5 & 0.584 & 0.0871 \\
\hline 0.53 & 0.738 & 0.07748 \\
\hline 0.56 & 0.779 & 0.06940 \\
\hline 0.6 & 0.823 & 0.06046 \\
\hline 0.63 & 0.876 & 0.05484 \\
\hline 0.67 & 0.928 & 0.4848 \\
\hline
\end{tabular}

The slot fill factor was considered during the optimization like one member has the values $a, c, H s 2$. The software calculates the slot area with slot liner and slot wedge $\left(\mathrm{T}_{\text {slotv }}\right)$ and without them too. Afterwards it calculates the maximum reachable slot fill factor.

$$
\begin{aligned}
& T_{\text {slot }}=\frac{a+c}{2} H_{s 2} \\
& k=0,9075 \frac{T_{\text {slot }}}{T_{\text {slot }}} \frac{T_{w}}{T_{w} E}
\end{aligned}
$$

where $k$ is the maximum slot fill factor, $T_{w E}$ is the area of the enamelled wire, $T_{w}$ is the effective area of the wire. The multiplier 0.9075 is the proportion of the areas of the circle and the hexagon.

After the software calculated the maximum slot fill factor it starts to determinate the number of wires per set with observance the number of turns $(N)$ and specifies the real slot fill factor:

$$
\begin{aligned}
& n w=\frac{T_{\text {slot }}}{2 T_{w H} N} \\
& k_{v}=\frac{\frac{T_{\text {slotV }}}{T_{w H}} \cdot T_{w}}{T_{\text {slot }}}
\end{aligned}
$$

where $n w$ is the number of wire per set $T_{w H}$ is the hexagonal area of the wire which was calculated from the area of the enamelled copper wire, $k_{v}$ is the real slot fill factor. The multiplier 2 comes from the double layer winding in eq. (10).

To the more precise value of the slot fill factor more simulations were made in the finite element software Infolytica to get the slot fill factor and wire diameters pairs. The fill factor was calculated for the different wire diameters and the results were compared. The simulations and the calculations were made for two different slot sizes, with double layer winding. The number of turns was 7 in both of the cases.

The software needed the value of the wire effective diameter and the number of wires per set. The table (see Table 2.) contains the wire sizes which were used during the comparison. 
Table 2. Diameters of the wires [11]

\begin{tabular}{|c|c|}
\hline \multicolumn{2}{|c|}{ Wire diameter } \\
\hline Effective & Enamelled \\
\hline 0.500 & 0.584 \\
\hline 0.530 & 0.738 \\
\hline 0.560 & 0.779 \\
\hline 0.600 & 0.823 \\
\hline 0.630 & 0.876 \\
\hline 0.670 & 0.928 \\
\hline 0.710 & 0.980 \\
\hline 0.750 & 1.032 \\
\hline 0.800 & 1.083 \\
\hline 0.850 & 1.145 \\
\hline 0.900 & 1.209 \\
\hline 0.950 & 1.271 \\
\hline 1.000 & 1.343 \\
\hline 1.060 & 1.415 \\
\hline 1.120 & 1.498 \\
\hline 1.180 & 1.600 \\
\hline 1.250 & 1.701 \\
\hline 1.320 & 1.804 \\
\hline 1.400 & 1.908 \\
\hline 1.500 & 2.012 \\
\hline 1.600 & 2.113 \\
\hline 1.700 & 2.237 \\
\hline 1.800 & 2.358 \\
\hline 1.900 & 2.482 \\
\hline 2.000 & 2.625 \\
\hline 2.120 & 2.778 \\
\hline 2.240 & 2.930 \\
\hline 2.360 & 3.132 \\
\hline & \\
\hline
\end{tabular}

The slot fill factor was calculated according to the eq. (9). The results are in Fig. 7. and Fig. 8 . 


\section{Slot fill factor - Slot area: $181,6 \mathrm{~mm}^{2}$}

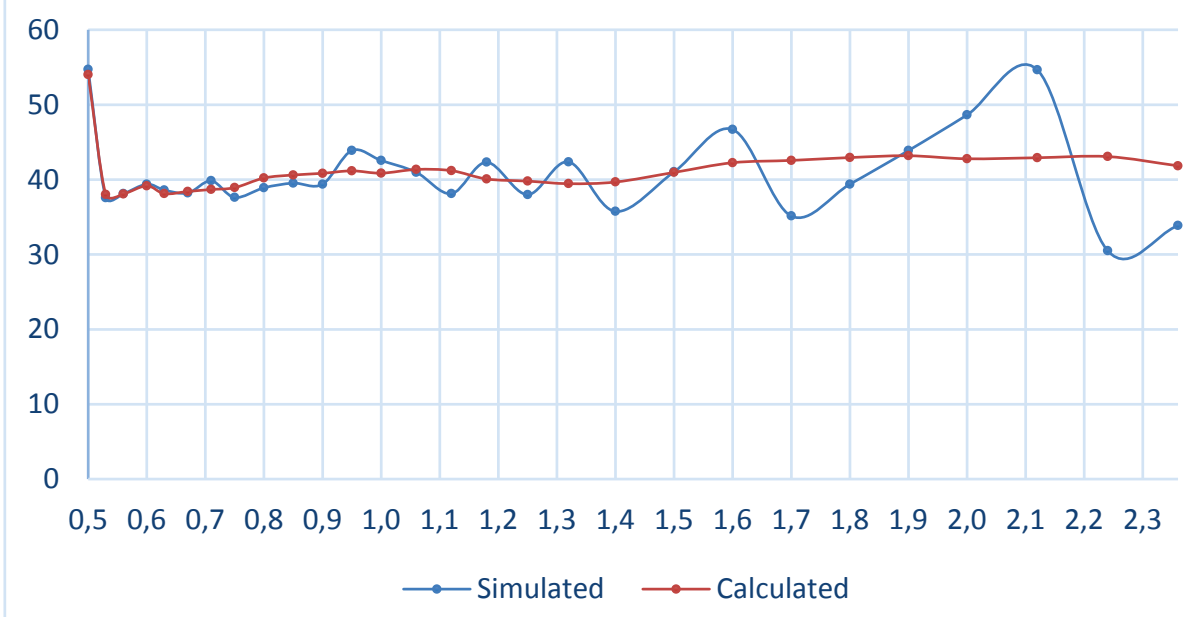

Figure 7. The slot fill factor results for the slot area $181,6 \mathrm{~mm}^{2}$

\section{Slot fill factor - Slot area: $220,5 \mathrm{~mm}^{2}$}

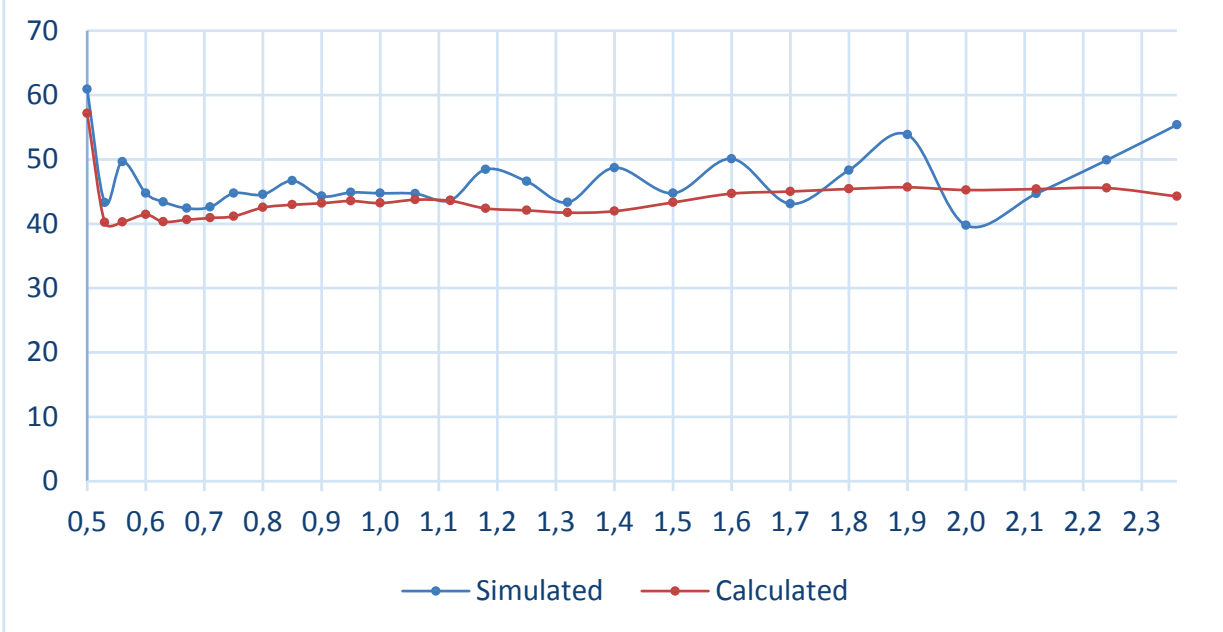

Figure 8. The slot fill factor results for the slot area $220,5 \mathrm{~mm}^{2}$

The calculated slot fill factor values are almost the same by the two slot sizes, the difference is marginal by the bigger slot size, the shapes of the curves are very similar. The simulated results are almost similar by the slot sizes but the main value of simulated fill factors by the bigger slot is $6 \%$ higher. 
It would be useful to fill the slot with double layer windings with the same number of turns by different wire diameters and complete these results with the real values.

\section{Conclusion}

In these researches the design was focused during the optimization to build the model correctly with the aware describing of the results. The optimization process varies the value of the parameters in wide ranges therefor it points out such kind of parameter combinations which were not expect during the design. The target was to make a general model of the outer rotor PMS motor and describe them prudently to use the model to give make good solutions for the different applications.

Later the optimization will be enlarged with better consideration the thermometric behaviour of the motor. The other plans are to complete the penalty function with the inducted voltage and to continue the investigation of the slot fill factor with check and complete the results with a real winding measurement.

\section{Acknowledgement}

The research work presented in this paper was carried out as part of the TÁMOP4.2.2.A-11/1/KONV-2012-0012 project in the framework of the New Széchenyi Plan. The realization of this project is supported by the European Union, and co-financed by the European Social Fund.

\section{References}

[1] Bittner F, Hahn I: Kriging-Assisted Multi-Objective Particle Swarm Optimization of Permanent Magnet Synchronous Machine for Hybrid an Electric Cars. IEEE International Electric Machines and Drives Conference, Chicago, IL, USA, 2013. DOI: $10.1109 /$ IEMDC.2013.6556123

[2] Duan Y, Harley RG: Method for Multi-Objective Design and Optimization of Three Phase Induction Machines. IEEE Transactions on Industry Applications, 2011.

DOI: $10.1109 /$ TIA.2011.2156372

[3] Geest Mvd, Polinder H, Ferreira JA, Zeilstra D:Optimization and Comparison of Electrical Machines using Particle Swarm Optimization. XXth International Conference on Electrical Machines, Marseille, 2012.

DOI: 10.1109/ICEIMach.2012.6350058

[4] Milot A, Korkosz M, Lukaniszyn M: Iron loss and eddy-current loss analysis in a low power BLDC motor with magnet segmentation. Archives of Electrical Engineering, 1. kötet, pp. 33-46, 2012.

DOI: $10.2478 / \mathrm{v} 10171-012-0003-5$

[5] Krotsch J, Piepenbreie B: Hybrid Algorithm for Multi-objective Optimization of PMSM using massively distributed Finite Element Analysis. 12th International Conference on Optimization of Electrical and Electronic Equipment, 2010. DOI: 10.1109/OPTIM.2010.5510429

[6] Kuslits M: Driving cycle based cost function for energetic optimization of PMS motors applied in electric vehicles. Workshop on Design, Simulation, 
Optimization and Control of Green Vehichles and Transportation, Györ, Hungary 2014.

[7] Jordan MI: Why the logistic function? A tutorial discussion on probabilities and neural networks. Computational Cognitive Science, Massachusetts Institute of Technology, 1995.

[8] Mclyman CWT: Transformer and inductor design handbook, NY, U.S.A.: Marcel Dekker, 2004.

DOI: $10.1201 / 9780203913598 . c h 8$

[9] Gieras JF: Permanent magnet motor technology: Design and applications, New York: MARCEL DEKKER Incorporated, 2002. ISBN: 0-8247-0739-7

[10] Szénásy I: Some actual questions at the development of up-to date PMSM motors. [Performance]. Workshop on Design, Simulation, Optimization and Control of Green Vehicles and Transportation, 2014.

[11] Specifications for particular types of winding wires - Part 0-1: General requirements - Enamelled round copper wire, IEEE Standard IEC 60317-0-1, 2013.

[12] Zhu ZQ, Howe D: Influence of design parameters on cogging torque in permanent magnet machines. IEEE Transactions on Energy Conversation, Vol. 15, No. 4, pp. 407-412, 2000.

DOI: $\underline{10.1109 / 60.900501}$

[13] Hanselman D: Brushless permanent motor design. Orono: MAGNA PHYSICS PUBLISHING, 1994. ISBN: 1-881855-15-5 